\title{
Drug or disease - Differentiating and managing motor symptoms associated with late onset Huntington's disease from extrapyramidal side-effects of antipsychotics
}

\author{
Seri Abraham ${ }^{1}$, Elspeth Guthrie ${ }^{2}$, Shahzada Nawaz ${ }^{3}$ and David Craufurd ${ }^{4}$
}

${ }^{1}$ ST6 General Adult Psychiatry, Lancashire Care NHS Foundation Trust, Pendleview Unit, Royal Blackburn Hospital, UK

${ }^{2}$ Professor of Psychosomatic Medicine and Consultant Psychiatrist, Greater Manchester Mental Health NHS Foundation Trust, Manchester Royal Infirmary, UK ${ }^{3}$ ST6 General Adult Psychiatry, Lancashire Care NHS Foundation Trust, Early Intervention Team, UK

${ }^{4}$ Senior Lecturer in Clinical Genetics, Manchester Centre for Clinical Genetic, St. Mary's Hospital, UK

\begin{abstract}
We discuss the case of a 59 year old gentleman with schizophrenia who presented with abnormal movements and severe weight loss. He was noted to have marked choreiform limb movements along with orofacial dyskinsea. He also had severe cognitive impairment with language difficulties. His abnormal movements were erroneously attributed to extra-pyramidal side effects from antipsychotic medication he had been prescribed for schizophrenia. Clinical suspicions of Huntington's disease prompted a genetic investigation which was positive for the disease. This led to input from specialist Huntington's services and the patient's physical health improved with robust multidisciplinary treatment. Greater awareness among health professionals regarding variability in disease presentation will improve early diagnosis and management of Huntington's disease.
\end{abstract}

\section{Background}

Huntington's disease is an autosomal dominant neurodegenerative condition which is progressive in nature [1]. It affects the basal ganglia initially and leads to cognitive, affective, motor and behavioural decline with the passage of time [2].

The classical motor disturbance associated with Huntington's disease is chorea. The prevalence rate is about $8-12$ per 100,000 in the Americas, Europe and Australia. However the rates are significantly lower in Asia and Africa, with an estimated prevalence of 0.5 per 100,000 in East Asian countries and even lower in African countries [3]. The condition affects both sexes equally with a mean age of onset of about 40 years. The age of onset ranges between 4 years to 80 years in the present literature [2].

The genetic basis for Huntington's disease is a mutation resulting CAG repeat expansion to $>35$ CAG sequence repeats in the gene which codes for a protein known as huntingtin present on the short arm of chromosome 4 [2]. In the general population, CAG sequence repeats range from 9-11 to 34-37 with an average of 17-20 [4]. Repeat expansion of more than 37 is strongly associated with the disease. Individuals with repeats of 36 to 39 may or may not develop Huntington's disease [4].

In terms of pathology of Huntington's disease, neuronal loss is predominantly seen in the striatal part of the basal ganglia and as the disease progresses this extends to the cerebral cortex [2]. Symptoms usually relate to localization of neurodegeneration in Huntington's disease. The different mechanisms postulated to explain neuronal death include induction of autophagy, induction of apoptosis, release of calcium from cells, transcriptional dysfunction and NMDA excitotoxicity [2].

Disease onset and progression depends on the number of CAG repeats. Studies have shown that the number of repeat expansion is inversely associated with age of onset of Huntington's disease [5]. Patients with more than 60 repeats usually present before the age of 20 (juvenile onset Huntington's disease) whereas patients with repeats of 36-38 present as late as 60 years or more (late onset Huntington's disease). Adult onset Huntington's disease presents around 40 years of age and has an average repeat sequence of about 44 [6].

Patients with adult onset Huntington's disease mainly present with chorea. Juvenile onset Huntington's disease patients present usually with rigidity associated with bradykinesia and rarely with seizures without chorea, late onset patients present with mild chorea, and, rarely, with psychiatric or cognitive difficulties $[7,8]$.

Psychiatric co-morbidities are seen in about $33 \%$ to $76 \%$ of patients depending on the methodology of the study [9]. Depression is the most common psychiatric co-morbidity and patients have a life time prevalence rate of about $39 \%$. Patients are also at four to six times more likely to commit suicide as compared with the general population [10]. Irritability, apathy, anxiety and obsessions are seen in patients with Huntington's disease. Psychotic symptoms similar to schizophrenia are seen in about $6 \%$ to $25 \%$ of the patients [11]. They are more common in juvenile onset Huntington's disease. Personality changes are the most common behavioural manifestation seen in patients with Huntington's disease [11].

Correspondence to: Seri Abraham, ST6 General Adult Psychiatry, Lancashire Care NHS Foundation Trust, Pendleview Unit, Royal Blackburn Hospital, Blackburn, BB2 3HH, UK, E-mail: seriabraham@hotmail.com

Received: January 07, 2017; Accepted: January 19, 2017; Published: January 21 2017 
We present the case of a middle aged gentleman with a history of schizophrenia, abnormal movements, and severe, life threatening weight loss. The abnormal movements had been mistakenly attributed to extra-pyramidal side-effects caused by his antipsychotic medication. This case illustrates the challenges in the diagnosis of Huntington's disease in patients with late onset of the condition, and those with psychiatric co-morbidities.

\section{Case presentation}

A 59 year old gentleman was admitted to a medical ward of a large teaching hospital in a state of severe malnutrition with a BMI of $13 \mathrm{~kg} / \mathrm{m}^{2}$. He had been living in a private psychiatric facility for a number of years, prior to his admission to the ward, with a diagnosis of schizophrenia. He was referred to the liaison psychiatry service for assessment of his mental state and abnormal movements.

On the ward, it became clear that the reason for his severe weight loss was due to difficulties with eating. He was struggling with coordinating his limb movements to enable him to get food into his mouth, and swallowing food without choking because of quite severe orofacial dyskinesia.

He had a history of schizophrenia and was being treated with an antipsychotic depot injection, namely pipothiazine palmitate. His abnormal movements had been attributed in the past to side-effects from his antipsychotic medication. The severity of his choreiform movements, however, was more suggestive of Huntington's disease and the psychiatric team organised testing for the disease, which came back positive. The depot was stopped after his admission to the ward.

The patient's physical status improved whilst he was on the ward and he gained weight. The nursing staff helped to feed him, as he was unable to feed himself. The medical team considered the possibility of a PEG because of his continuing difficulties with eating, however, this was not required as his dietary intake and weight improved significantly, with basic nursing support.

The patient was noted to be cognitively impaired, with severe loss of short-term memory. He was not able to cooperate with formal cognitive testing. He was unable to retain information, and could not remember any conversations, even if they had occurred only a few minutes earlier. He could not recognise or remember any members of staff. He knew that he was in hospital but was disorientated for time and person. He could answer simple questions although he found it difficult to articulate some words due to his dysarthria. His mood appeared to be euthymic and improved as his physical status improved. He had no psychotic phenomena and he was appreciative of the care that he was receiving.

He was started on Olanzapine $5 \mathrm{mg}$ b.d. which we advised to continue for the foreseeable future. Based on the records from his previous placement, it appeared that the patient was estranged from his family and apparently his mother had specifically requested no further contact with him after she was subject to an assault from him some years previously. In relation to other potential family members, with regards to Huntington's disease, it was difficult to be clear from him, the exact nature of his family and history. He told us that his father was dead but would not discuss the circumstances of his death. He told us on occasions that he had a sister and on other occasions that she was dead. He also told us that he had a brother but he was unaware of his address.

\section{Investigations}

The patient's genetic testing revealed that an allele with 44 CAG repeats. CT head showed generalized atrophic changes within the brain, significantly greater than expected for the patient's age. The patient's routine blood results and ECG were normal.

\section{Differential diagnosis}

This patient presented with chorea which is a classical motor sign of Huntington's disease and hence this was the primary differential diagnosis. Other differential diagnoses that were considered were Sydenham's chorea, Wilson's disease, spinocerebellar ataxia, vitamin B 12 deficiency and syphilis. Input from neurology and relevant investigations helped rule out the conditions mentioned above. The patient's cognitive deficits are best explained by a dementia-like illness secondary to advanced Huntington's disease.

\section{Treatment}

The patient's depot typical antipsychotic was stopped and he was started on an oral atypical antipsychotic medication. The patient's anticholinergic medication which he had been prescribed prior to hospital admission for extra-pyramidal side effects was stopped.

\section{Outcome and follow-up}

The patient was referred to a specialist Huntington's clinic for further follow-up and was discharged from the Liaison Psychiatry service back to the nursing home he was placed in, with specific advice about his need for help with feeding. The patient is currently placed in a specialist neuro-rehabilitation unit. The patient was reviewed again in May 2016. His movements and cognition was more or less unchanged from his initial presentation. However his BMI has significantly improved and overall, appeared physically, to be in a better shape. $\mathrm{He}$ continues to receive input from specialist Huntington's disease team and local mental health services.

\section{Discussion}

The unusual features of this case include: the late diagnosis of Huntington's disease; the diagnostic overlapping with paranoid schizophrenia; the patient's extreme cachexia and staff's misattribution of his abnormal movements to extrapyramidal side-effects related to antipsychotic use rather than to motor symptoms of Huntington's disease.

It is likely that the patient's Huntington's disease was of late onset as an individual with 40-50 CAG repeats generally has a mid-life onset $[6]$.

Weight loss is considered to be an integral manifestation of the disease, especially in late onset cases although the factors causing weight loss in patients with Huntington's disease is not completely understood. Weight loss has been noted to be secondary to a hypermetabolic state and is proportional to the number of CAG repeats [12].

Hyperkinesia is not considered to be responsible for weight loss as even patients on a high calorie diet show significant weight loss [13]. However, the cachexia in our patient was explained by a failure to diagnose Huntington's and a lack of understanding about his inability to feed himself because of his choreiform movements. Food would be placed in front of the patient however he would struggle with co-ordinating movements due to the chorea to place the food in his mouth and this was further complicated by orofacial dyskinesia. He was literally starving to death. His BMI improved on the ward with proactive nursing care and he enjoyed and ate all the food that was 
provided for him.

Antipsychotic induced orofacial dyskinesia is usually considered, together with tardive dyskinesia, as part of a wider problem related to extra-pyramidal side-effects of antipsychotic medication (EPSE). Orofacial dyskinesia is characterized by involuntary and repetitive movements in the oral, lingual and buccal area. It is usually nonreversible and has a prevalence rate of about $24 \%$ in a review conducted in 1988 [14]. However with the advent of newer atypical antipsychotics, a 2.5 fold reduction has been noted and the incidence rate at present is around $2 \%$ [15].

The motor movement classically associated with Huntington's disease is chorea and is derived from the Greek word for 'dance'. It is defined as a rapid, unpredictable, involuntary, non-repetitive movement affecting limbs, trunk and face [16]. Patients also present with other abnormal movements, difficulty in initiating movements and rigidity. Dystonia leading to abnormal postures tics similar to that seen in Tourette syndrome and cerebellar signs, mainly ataxia, are also seen in patients with Huntington's disease [16].

This results in difficulties with activities of daily living such as maintaining self-hygiene and self-caring. Abnormal movements associated with Huntington's disease are usually non-repetitive and pure oro-facial movements are less common than in tardive dyskinesia. Conversely, severe choreiform movements are characteristics of Huntington's disease and much less common in EPSE [13]. However there is little information regarding the risk of tardive dyskinesia in Huntington's patients who are prescribed antipsychotic medication. Antipsychotics help in reducing the intensity of chorea in patients with Huntington's disease due to their anti-dopaminergic action. Haloperidol, pimozide, clozapine, olanzapine, aripirazole, risperidone and quetiapine are antipsychotics that have been shown to help manage chorea [17].

Cognitive deficits secondary to Huntington's disease are typically subcortical in nature. Patients generally present with impaired cognition, attention deficits, impaired problem solving, visuospatial problems and constructional abilities [18]. However as the disease progresses, patients have been noted to have cortical features as well such as prominent memory impairment, language difficulties and impairment in executive functions [19]. The patient's marked cognitive problems were most likely indicative of the severity of his Huntington's disease.

We believe that greater awareness among health professionals of Huntington's disease, especially when symptoms overlap with those of other psychiatric co-morbidities, will improve diagnosis and management of similar patients.

\section{Key learning points}

1. The age of onset and clinical presentation of Huntington's disease is largely dependent on the number of trinucleotide repeats. As a result, an atypical presentation could lead to diagnostic uncertainty.

2. Presentation in patients with Huntington's disease may overlap with other comorbid psychiatric conditions, often causing difficulties in diagnosis and management.

3. Generally, antipsychotics help to improve abnormal movements rather than exacerbating them.

4. The nature of cognitive impairment in patients with Huntington's disease is an important marker with regards to disease progression.
5. Greater awareness among health professionals about Huntington's disease will improve diagnosis and management of patients.

\section{References}

1. Albin RL, Tagle DA (1995) Genetics and molecular biology of Huntington's disease Trends Neurosci 18: 11-14. [Crossref]

2. Reiner A, Dragatsis I, Dietrich P (2011) Genetics and neuropathology of Huntington's disease. Int Rev Neurobiol 98: 325-372. [Crossref]

3. Walker FO (2007) Huntington's disease. Lancet 369:118-218.

4. Read AP (1993) Huntington's disease: testing the test. Nat Genet 4: 329-330. [Crossref]

5. Andrew SE, Goldberg YP, Kremer B, Telenius H, Theilmann J, et al. (1993) The relationship between trinucleotide (CAG) repeat length and clinical features of Huntington's disease. Nat Genet 4: 398-403. [Crossref]

6. Margolis RL, Ross CA (2003) Diagnosis of Huntington disease. Clin Chem 49: 17261732. [Crossref]

7. McNeil SM, Novelletto A, Srinidhi J, Barnes G, Kornbluth I, et al. (1997) Reduced penetrance of the Huntington's disease mutation. Hum Mol Genet 6: 775-779. [Crossref]

8. Ribaï P, Nguyen K, Hahn-Barma V, Gourfinkel-An I, Vidailhet M, et al. (2007) Psychiatric and cognitive difficulties as indicators of juvenile huntington disease onset in 29 patients. Arch Neurol 64: 813-819. [Crossref]

9. Roos RA (2010) Huntington's disease: a clinical review. Orphanet J Rare Dis 5: 40 [Crossref]

10. Rosenblatt A, Leroi I (2000) Neuropsychiatry of Huntington's disease and other basa ganglia disorders. Psychosomatics 41: 24-30. [Crossref]

11. Cummings JL (1995) Behavioral and psychiatric symptoms associated with Huntington's disease. Adv Neurol 65: 179-186. [Crossref]

12. Aziz NA, van der Burg JM, Landwehrmeyer GB, Brundin P, Stijnen T; EHDI Study Group, et al. (2008) Weight loss in Huntington disease increases with higher CAG repeat number. Neurology 71: 1506-1513. [Crossref]

13. Harper PS (1991) Huntington's disease (Major problems in Neurology), Saunders Ltd.

14. Kane JM, Woerner M, Lieberman J (1988) Tardive dyskinesia: prevalence, incidence, and risk factors. J Clin Psychopharmacol 8: 52S-56S. [Crossref]

15. Lerner PP, Miodownik C, Lerner V (2015) Tardive dyskinesia (Syndrome): Current concept and modern approaches to its management. Psychiatry Clin Neurosci 69: $321-334$

16. Casaca-Carreira J, Temel Y, van Zelst M, Jahanshahi A (2015) Coexistence of Gai Disturbances and Chorea in Experimental Huntington's Disease. Behav Neurol 2015 970204. [Crossref]

17. Videnovic A (2013) Treatment of huntington disease. Curr Treat Options Neurol 15 424-438. [Crossref]

18. Salmon DP, Heindel WC (1998) The Effects of Cortical and Subcortical Brain Dysfunction on the Cognitive Presentation of Dementing Disorders. NeuroScience News 1: 36-42.

19. Peavy GM, Jacobson MW, Goldstein JL, Hamilton JM, Kane A, et al. (2010) Cognitive and functional decline in Huntington's disease: dementia criteria revisited. Mov Disord 25: 1163-1169. [Crossref]

Copyright: (C2017 Abraham S. This is an open-access article distributed under the terms of the Creative Commons Attribution License, which permits unrestricted use, distribution, and reproduction in any medium, provided the original author and source are credited. 